\title{
An improved energy consumption method for wireless sensor networks
}

\author{
Seyedeh Zahra Yazdanpanah ${ }^{a^{*}}$, Yousef Abbasnejad Varzi ${ }^{a}$, Ali Haronabadic and Seyed Javad \\ Mirabedinic
}

${ }^{a}$ Department of Computer Science, International Kish Branch, Islamic Azad University, Kish, Iran

${ }^{b}$ Faculty of Computer Science, Central Tehran Branch, Islamic Azad University, Kish, Iran

${ }^{c}$ Faculty Member, Department of Computer Science, Central Branch, Islamic Azad University, Tehran, Iran

\section{H R O N I C L E}

Article history:

Received December 2, 2013

Accepted 8 May 2014

Available online

May 152014

Keywords:

Energy efficiency

Network longevity

Heterogeneous network

\section{A B S T R A C T}

This paper presents a new basic clustering algorithm to increase the wireless sensor networks energy and longevity, efficiently. In each period, cluster heads are selected based on the maximum energy and the minimum distance through the nodes. Therefore, the protocol to obtain good performance in terms of longevity is achieved by balancing the energy load among all nodes. The simulation results obtained from the MATLAB software indicate that the suggested approach is effective in prolonging the network longevity.

\section{Introduction}

The wireless sensor networks (WSNs) normally consists of many sensor nodes, which are often used to monitor the environmental and physical conditions, such as temperature, sound, pressure, etc. and they dispatched the necessary data through the network to a base station (BS). The base station usually applies nodes with high processing power, high storage capacity and re-chargeable battery. Data are gathered in the sensor nodes and they are sent to the base station directly or via other nodes. All collected nodes are processed in the base station for one specific parameter such as temperature, pressure, moisture, etc. and the expected value from that parameter is measured (Akyildiz et al., 2002). The WSN communication protocols performance influences the energy by equal distribution and the reduction in energy consumption entails the prolongation of the network longevity. Therefore, an efficient energy protocol plays essential role in design perspectives (Braginsky \& Estrin, 2002). In such protocol, the sensor nodes are grouped into a set of clusters where each cluster has a leader

*Corresponding author.

E-mail addresses: S59 yazdanpanah@yahoo.com (S. Z. Yazdanpanah) 
called the head cluster $(\mathrm{CH})$ and the nodes in one cluster collect their data directly and transfer it to the base station.

In this paper, we present a new approach based on the nodes primary energy and the distance between them in order to provide a chance for the closer node to be selected as the cluster head. Simulation results show that the suggested protocol, compared with the LEACH protocol, is effective in prolonging the network longevity.

\section{Framework}

Hierarchical clustering in wireless sensor networks can significantly influence on the scalability of the whole system, longevity, and energy efficiency. The hierarchy routing is an efficient technique towards minimization of energy consumption contained in a cluster and the integration and combination of data towards reducing the number of messages sent to the base station. Hierarchical clustering is especially efficient in different applications, which require hundreds or thousands of nodes. The scalability makes the request to load balancing and proper exploitation urgent. In addition, supporting the network scalability as well as reducing the energy consumption and clustering improve the relative efficiency of the method. In addition, it can maintain the communication bandwidth; since it limits the intra-clustering interactions domain to the cluster heads and prevents redundancy in exchanging messages among the sensor nodes. Clustering can also stabilize the network topology on the level of sensors and reduce the overhead and the total cost of the topology maintenance.

\section{Related works}

There are literally numerous routing algorithms introduced for the sensor networks and the routing protocols in the sensor networks can be classified into two main groups of the network structure, namely plain and hierarchical. In plain routing protocols, there is not such concept as the leader node and all nodes are the same. Some of these methods include for Spin (Levis et al., 2004), rumor (Akyildiz et al., 2002), Directed Diffusion (Tilak et al., 2002), EBRP (Sarrafzadeh et al., 2006), GBR (Jafari et al., 2006), EAR (Amini et al., 2007), GEAR (Sun, 2001), SPEED (Nasipuri, 2004).

In hierarchical routing protocols, nodes are classified and some nodes bearing more energy compared with others are selected as the leaders such as LEACH (Zhang \& Lee, 2000) LEACH (Mamatha \& Sharma, 2010) TEEN (Dastgheib et al., 2011) MECN (Zadeh, 1965) HDAR (Celikyilmaz \& Türksen, 2009). Data-centered protocols, protocols based on location information, and the protocols are other types of routers. In data-centered protocols, the central station transmits a query to a specific area and waits to get data from the nodes placed in the desired area. Examples of these sets are: SPIN, Rumor, Directed Diffusion, GBR, TEEN, COUGAR (Biswas, 2010).

There are also routing protocols based on the location information used for sending data to the desired areas (e.g. MECN, GAF, GEAR and SPEED). In routing protocols aware of the energy, the main focus is on the efficient utilization of energy to increase the network longevity. Examples of these sets are EAR, EBRP, (Ryan Florin) SELAR. In some papers, the routing protocols are classified into three sets, namely proactive, reactive and hybrid, depending on how the transmitter detects a path towards the receiver. In proactive protocols, all paths are calculated before even they are requested, while in reactive protocols, paths are calculated only when they are required. The hybrid protocols are a combination of the two previous protocols. According to Hincapié et al. (2006) and Heidemann et al. (2002), multi-path routing protocols are also used in such a way that numerous paths are established between the source node and the central node and if the desired path is broken, another path is used to establish a connection. 


\section{The suggested protocol}

\subsection{The effect of energy levels on cluster head selection}

Let $\mathrm{N}$ be the number of sensor nodes distributed evenly inside the $\mathrm{M} \times \mathrm{M}$ region. The network is organized into the cluster hierarchy and the cluster head is produced by the sensor nodes inside the clusters in order to reduce the correlated data. The cluster head directly transmits the collected data to the base station. In the heterogeneous networks, there are three kinds of sensor nodes, namely advanced nodes, normal nodes, and intermediate nodes. Let $m$ be a fraction of the whole node, which has more local energy time $\alpha$ than the normal nodes, which is the advanced nodes, and $b$ is a fraction of the whole node $n$, which contains more local energy time $\beta$ than the normal nodes that is the intermediate node. The total primary energy associated with the heterogeneous networks in all three levels is as follows,

$n \cdot(1-m-b) \cdot E_{0}+n \cdot m \cdot E_{0} \cdot(1+\alpha)+n \cdot b \cdot E_{0} \cdot(1+\beta)=n \cdot E_{0} \cdot(1+\alpha \cdot m+b \beta)$

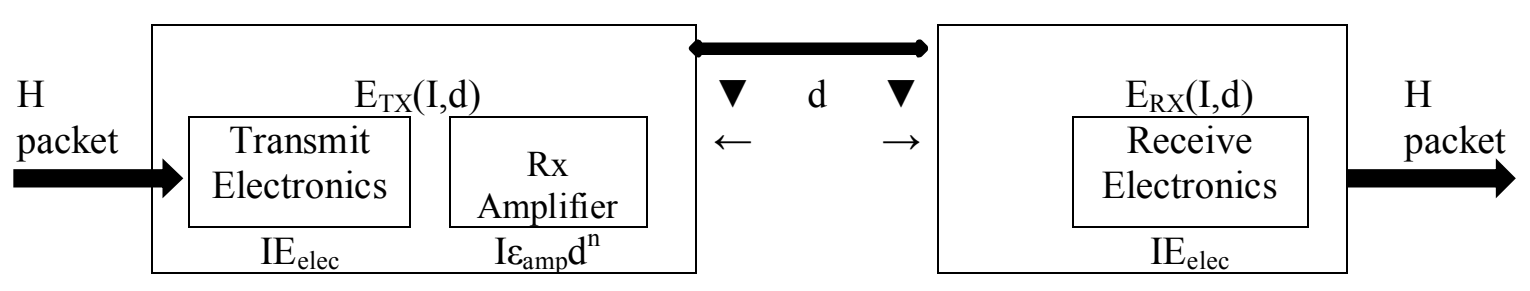

Fig. 1. The radio energy dissipation model

Based on the radio energy dissipation model suggested by Heinzelman et al. (2000) and to achieve suitable signal-to-noise ratio (SNR) in L-bit message transmission, the energy consumed by the radio is calculated as follows,

$$
E_{T x}(l, d)= \begin{cases}L \cdot E_{\text {elec }}+L \cdot \epsilon_{f s} \cdot d^{2} & \text { if } d \leq d_{0} \\ L . E_{\text {elec }}+L . \epsilon_{m p} \cdot d^{4} & \text { if } d>d_{0}\end{cases}
$$

where $\mathrm{E}_{\text {elec }}$ is the consumed energy per bit executing $\mathrm{E}_{\mathrm{Tx}}$ with transmitter or receiver of the circuit $\epsilon_{f s} d^{2}$ and $\epsilon_{m p} d^{4}$ in which the transmitter amplifier model is used, $d$ is the distance between the transmitter and receiver. The energy required for receiving the radio messages is as follows,

$\mathrm{E}_{\mathrm{Rx}}(l)=l E_{\text {elec }}$

Considering the radio energy dissipation model, the minimum energy required is proportionate to the square of the distance between the transmitter to the receiver and to the destination (Qi et al., 2002). As the distance increases, the energy consumption increases, significantly. This means that the cluster heads far from the base station should consume more energy to transmit the data than the ones, which are closer to the base station. Therefore, there is a significant difference between the energy consumption rate of the nodes near the base station and those, which are far from the base station. In our method, the nodes with less distance and the nodes with more distance from the base station have the least chance to be selected as the cluster head in the two current periods. After the base station has been deployed, it sends a 'Welcome' message to all the nodes in a certain power level. Each node can calculate the approximate distance $D_{i}$ to the base station based on the received signal power. Therefore, we introduce new probabilities for each type of nodes according to the primary energy and the distance between each node and the base station, which is in favor of the nodes with more power and those nodes that are closer to the base station in order to become the cluster heads. 
Each normal node becomes the cluster head one time every $\frac{1}{P_{\text {opt }}}(1+\alpha \cdot m+b \cdot \beta)$.

$P_{n r m}=\frac{P_{o p t}}{1+a \cdot m+b \cdot \beta}$

Each intermediate node becomes the cluster head one time every $\frac{1}{P_{o p t}}(1+\alpha \cdot m+b \cdot \beta$.

$P_{a d v}=\frac{P_{o p t} *(1+a)}{1+a \cdot m+b \cdot \beta}$

Each advanced node becomes the cluster head one time every $\frac{1}{P_{o p t}}(1+\alpha \cdot m+b . \beta$.

$P_{\text {int }}=\frac{P_{\text {opt }} *(1+\beta)}{1+a \cdot m+b \cdot \beta}$

where,$P_{\text {opt }}$ is the number of optimum cluster heads that is always a number less than 1 and the denominator is a number greater than 1 . There is this guarantee that this equation yields a number less than 1 , which is a desired value. It should be noted that no matter which interval $\alpha$ and $\beta$ are considered in, the denominator is always less than the numerator.

\subsection{The effect of the distance on the cluster head selection}

In this step, we include the energy and distance. The probabilities for changing into the cluster head are as follows: if $D_{i}$ is the distance between the node $S_{i}$ and the base station, and $D_{\text {avg }}$ is the mean distance between the nodes and the synch; distance $D_{\mathrm{i}} \leq \mathrm{D}_{\text {avg }}$ is calculated as follows,

$$
\begin{aligned}
& P_{n r m}=\frac{P_{o p t}}{(1+a m+b \beta)} *\left(1-\frac{D_{i}}{D_{a v g}}\right) \\
& P_{\text {int }}=\frac{(1+\beta) P_{o p t}}{(1+a m+b \beta)} *\left(1-\frac{D_{i}}{D_{a v g}}\right) \\
& P_{a d v}=\frac{(1+\alpha) P_{o p t}}{(1+a m+b \beta)} *\left(1-\frac{D_{i}}{D_{a v g}}\right) \\
& P_{n r m}=\frac{P_{o p t}}{(1+a m)} \\
& P_{a d v}=\frac{(1+\alpha) P_{o p t}}{(1+a m)}
\end{aligned}
$$

and $\mathrm{D}_{\mathrm{avg}}$ is calculated as follows,

$D_{a d v}=\frac{1}{N} \sum_{i=1}^{N} D_{i}$

\subsection{The appropriate selection threshold for becoming a cluster head}

The value $T(s n)$, i.e. the threshold for selecting the cluster head, is used to normalize the nodes in each round for normal nodes, $T(s a)$ for advanced nodes, and $T(s i)$ for intermediate nodes that are obtained as follows: 


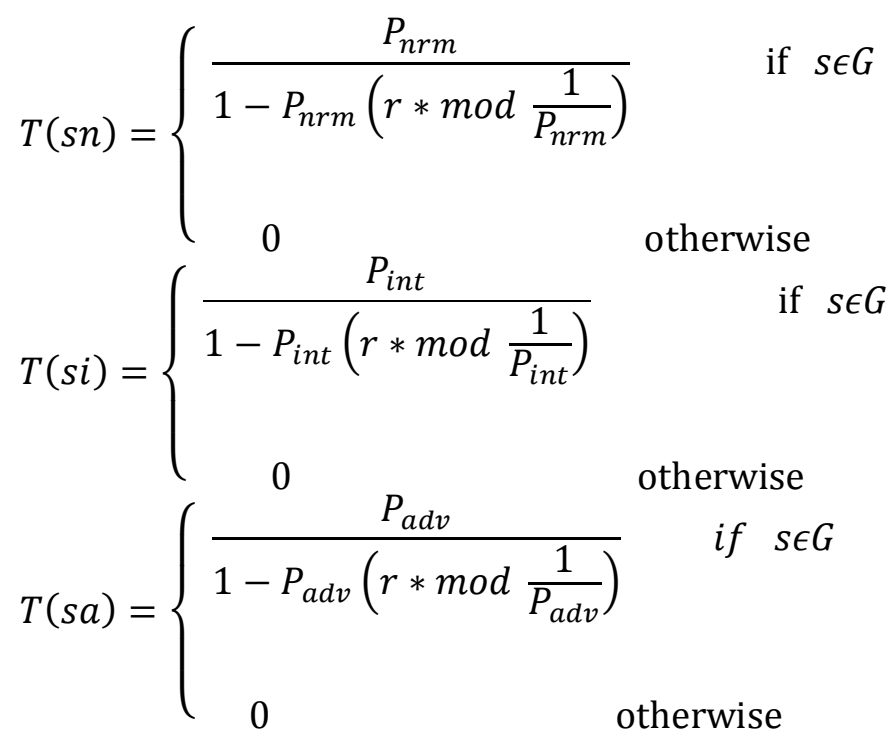

where $r$ is the current round, $G$ is a set of nodes to become the cluster head.

\subsection{The relationships between the cluster head and the members}

As for LEACH, the cluster head propagates its status to all sensor nodes after the cluster head is chosen. Then, other nodes choose the most appropriate cluster head (normally, the closest cluster head) and organize themselves. Next, the cluster members are generated based on the TDMA software and transmit their data to the cluster head.

\subsection{The relationship between the cluster head and the synch}

Each node transmits its data to the cluster head in a specified time. The cluster head node keeps all information associated with the nodes in its cluster. After the data has been received, the cluster head node applies the signal processing functions for data compression. Each cluster head can synchronize the collected data. The energy consumed by the cluster head consists of three parts: information reception, data collection, and data transmission.

$E\left(C H_{i}\right)=m_{i} l E_{\text {elec }}+\left(m_{i}+1\right) l E_{D A}+l\left(E_{\text {elec }}+\epsilon_{f s} d^{2}\right)$

where $m_{i}$ is the total members in a cluster, $d=D_{i}$ is the distance between $C H$ and BS. In this mode: $\mathrm{d}<\mathrm{d}_{0}$.

\section{The simulation results}

The evaluation of the protocol performance is executed based on the MATLAB software. We consider a wireless sensor network with $\mathrm{N}=100$ nodes that is randomly distributed in a $200 \times 200$ field. The distance between the network nodes and the base station equals $50 \mathrm{~m}<d t o B S<282.8 \mathrm{~m}$. The radio parameters used in our simulation are shown in Table 1.

$$
K_{\text {opt }}=\frac{\sqrt{100}}{\sqrt{2 \pi}} \sqrt{\frac{10}{0.0013}} \frac{400}{\left(50 m<d_{\text {toBS }}<282.2 m\right)^{2}} \quad \rightarrow \quad 2<K_{\text {opt }}<55
$$


Table 1

The radio features used in our simulation

\begin{tabular}{cc}
\hline Parameters & Value \\
\hline Network range & $(-2006-200)$ to $(200$ (200) \\
$N$ & 100 \\
$d_{0}$ & $87.7 \mathrm{~m}$ \\
Base station location & $(100,100)$ \\
$E_{\text {elec }}$ & $50 \mathrm{nj} / \mathrm{bit}$ \\
$\varepsilon_{f s}$ & $10 \mathrm{nj} / \mathrm{bit} / \mathrm{m}^{2}$ \\
$\varepsilon_{m p}$ & $0.0013 \mathrm{nj} / \mathrm{bit} / \mathrm{m}^{4}$ \\
$p_{\text {leach }}$ & 0.05 \\
Number of time frames in each round & 1 \\
Length of each time frame & $20 \mathrm{~s}$ \\
$E_{D A}$ & $5 \mathrm{nj} / \mathrm{bit} / \mathrm{signal}$ \\
Primary energy for each node & $0.5 \mathrm{j}$ \\
Packet size & $500 \mathrm{bytes}$ \\
\hline
\end{tabular}

\subsection{The system longevity in the executive period}

Fig. 2 shows that the present nodes in our suggested protocol continue to stay for longer time. Therefore, we can understand that by increasing the number of heterogeneous nodes in the protocol, we can be hopeful about an improvement in nodes longevity. This comes from the fact that the energy heterogeneity leads to the selection of cluster heads from among these nodes and helps the energy balance. The constant metric allows the user to have reliable information about the sensing area. This reliability is very important for sensitive applications such as tracking a jungle fire, which indicates that the suggested protocol worked better.

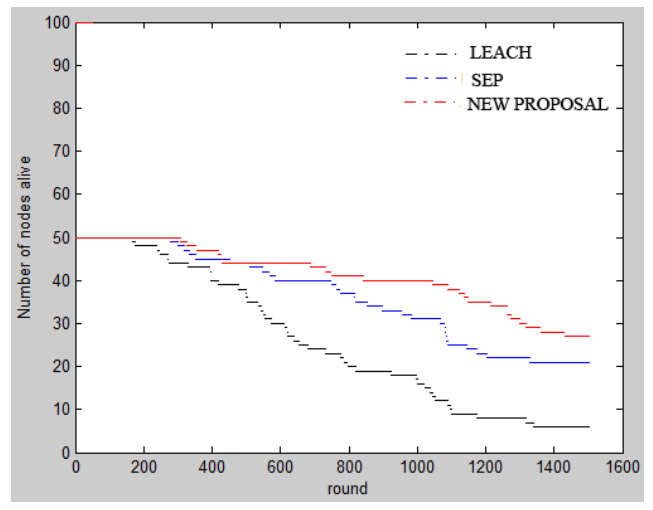

Fig. 2. The system longevity while executing the protocols

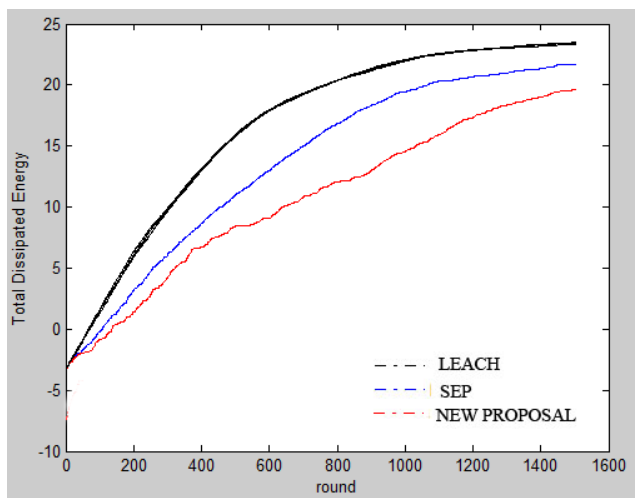

Fig. 3. Total dissipated energy while executing the protocols

\subsection{The energy consumption rate during the period}

Fig. 3 shows the total energy consumption rate in the network as compared to the time. According to the simulation results, we understand that using the suggested protocols lead to an economization in the amount of energy consumed by the network. This reduction in the total consumed energy in the network is associated with the significant reduction of the redundant transmissions. The suggested protocol has lower energy consumption rate compared with other protocols and this leads to an increase in the network longevity. Since our approach is to consider the distance between the nodes and the synch, the cluster head far away from the base station has more energy consumption 
compared to the cluster head news the base station. Our approach led to an economization in the total network energy.

\subsection{The number of packets sent during the period}

It can be seen from the results of Fig. 4 and Fig. 5 that the proposed protocol sends the packets to the base station with lower energy. Also during the periods, more packets are supported. Since the cluster heads are selected optimally, their redundant transmission rate is lower, their longevity is increased, and of course they support more transmitting packets.

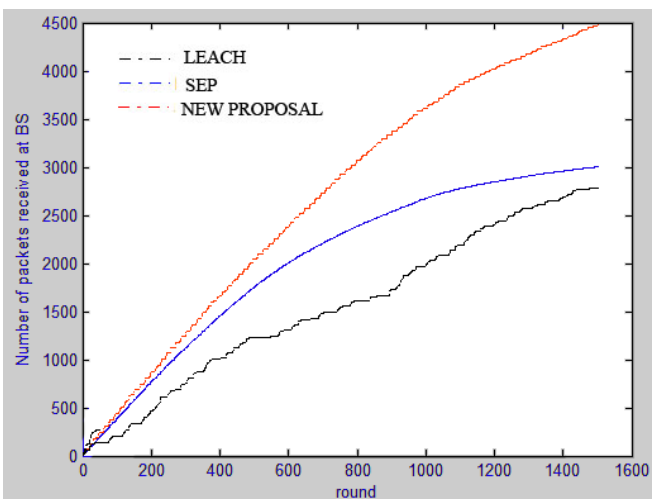

Fig. 4. The number of packets sent to BS in each round while executing the protocol

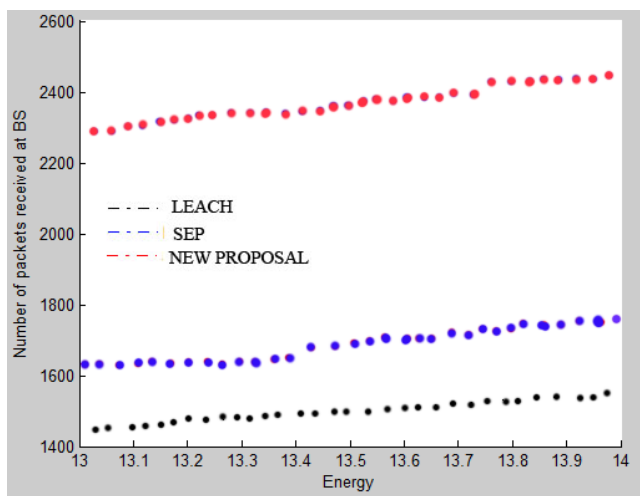

Fig. 5. The number of packets sent to BS in each ronud after excecuting the protocol

\subsection{The FND and HNA criteria}

In order to accurately compare the effective network longevity, the presented criteria, namely FND and HNA were considered here. Fig. 6 and Fig. 7 show these criteria for the simulation.

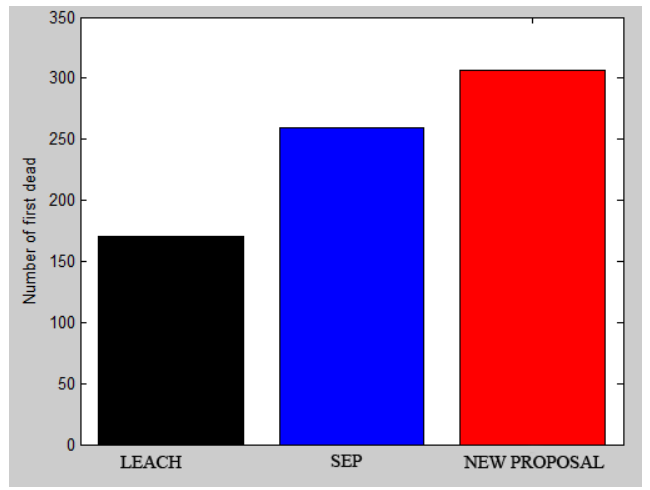

Fig. 6. A comparison of the first dead node while executing the protocol

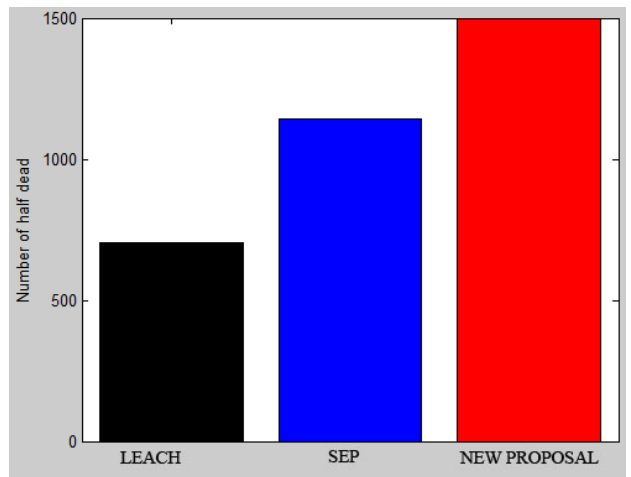

Fig. 7. A comparison of the half-dead nodes while executing the protocol

As seen in the above figures, considering the optimum selection of the cluster head, we reduced the number of redundant transmissions and were able to inprove these two criteria accordingly.

We have in mind that in the obrined simulation in the optimum mode, the advanced node turn into the cluster head more than the intermediate nodes and the intermediate nodes do the same more than the normal nodes. This helps maintain the nodes that are distributed evenly among the sensors during the periods. 


\section{Conclusion}

In this paper, we have presented a protocol used in the heterogenous wireless sensor networks compared with the LEACH protocol. For the suggested protocol, the sensor node selects itself indepedently as a cluster head based on its primary energy and its distance from the base station. Therefore, the nodes close to the base station containing more energy than other nodes have more chance to be selected as a cluster head. The results obtained from the simulation show that the suggested protocol has better performance in exploiting the energy and the network longevity.

\section{Acknowledgement}

The authors would like to thank the anonymous referees for constructive comments on earlier version of this paper.

\section{References}

Akyildiz, I. F., Su, W., Sankarasubramaniam, Y., \& Cayirci, E. (2002). A survey on sensor networks. Communications magazine, IEEE, 40(8), 102-114.

Akyildiz, I. F., Su, W., Sankarasubramaniam, Y., \& Cayirci, E. (2002). Wireless sensor networks: a survey. Computer networks, 38(4), 393-422.

Amini, N., Miremadi, S. G., \& Fazeli, M. (2007, April). A hierarchical routing protocol for energy load balancing in wireless sensor networks. In Electrical and Computer Engineering, 2007. CCECE 2007. Canadian Conference on (pp. 1086-1089). IEEE.

Biswas, N. (2010). Leader Election in Mobile Ad-Hoc Network. Bengal University.

Braginsky, D., \& Estrin, D. (2002, September). Rumor routing algorthim for sensor networks. In Proceedings of the 1st ACM international workshop on Wireless sensor networks and applications (pp. 22-31). ACM.

Celikyilmaz, A., \& Türksen, I. B. (2009). Modeling uncertainty with fuzzy logic: with recent theory and applications (Vol. 240). Springer.

Chen, Y., \& Zhao, Q. (2005). On the lifetime of wireless sensor networks.Communications Letters, IEEE, 9(11), 976-978.

Chlamtac, I., Conti, M., \& Liu, J. J. N. (2003). Mobile ad hoc networking: imperatives and challenges. Ad Hoc Networks, 1(1), 13-64.

Chen, M., Kwon, T., \& Choi, Y. (2006). Energy-efficient differentiated directed diffusion (EDDD) in wireless sensor networks. Computer Communications,29(2), 231-245.

Dastgheib, S. J., Oulia, H., \& Ghassami, M. R. S. (2011, December). An efficient approach for clustering in wireless sensor network using fuzzy logic. InComputer Science and Network Technology (ICCSNT), 2011 International Conference on (Vol. 3, pp. 1481-1486). IEEE.

Florin, R. (2011). A penalty-based approach to handling cluster sizing in mobile ad hoc networks (Doctoral dissertation, Old Dominion University).

Handy, M. J., Haase, M., \& Timmermann, D. (2002). Low energy adaptive clustering hierarchy with deterministic cluster-head selection. In Mobile and Wireless Communications Network, 2002. 4th International Workshop on (pp. 368-372). IEEE.

Heidemann, J., Silva, F., Intanagonwiwat, C., Govindan, R., Estrin, D., \& Ganesan, D. (2001, October). Building efficient wireless sensor networks with low-level naming. In ACM SIGOPS Operating Systems Review (Vol. 35, No. 5, pp. 146-159). ACM.

Hincapié, R. C., Correa, B. A., \& Ospina, L. (2006). Survey on clustering Techniques for mobile ad hoc networks. IEEE, Los Alamitos.

Heinzelman, W. R., Kulik, J., \& Balakrishnan, H. (1999, August). Adaptive protocols for information dissemination in wireless sensor networks. InProceedings of the 5th annual ACM/IEEE international conference on Mobile computing and networking (pp. 174-185). ACM. 
Heidemann, J., Silva, F., \& Estrin, D. (2003, November). Matching data dissemination algorithms to application requirements. In Proceedings of the 1st international conference on Embedded networked sensor systems (pp. 218-229). ACM.

Heidemann, J., Silva, F., Yu, Y., Estrin, D., \& Haldar, P. (2002). Diffusion filters as a flexible architecture for event notification in wireless sensor networks. Technical Report ISI-TR-556, USC/Information Sciences Institute.

Heinzelman, W. R., Chandrakasan, A., \& Balakrishnan, H. (2000, January). Energy-efficient communication protocol for wireless microsensor networks. InSystem Sciences, 2000. Proceedings of the 33rd Annual Hawaii International Conference on (pp. 10-pp). IEEE.

Intanagonwiwat, C., Govindan, R., Estrin, D., Heidemann, J., \& Silva, F. (2003). Directed diffusion for wireless sensor networking. Networking, IEEE/ACM Transactions on, 11(1), 2-16.

Jafari, R., Encarnacao, A., Zahoory, A., Dabiri, F., Noshadi, H., \& Sarrafzadeh, M. (2005, July). Wireless sensor networks for health monitoring. In Mobile and Ubiquitous Systems: Networking and Services, 2005. MobiQuitous 2005. The Second Annual International Conference on (pp. 479481). IEEE.

Levis, P., Madden, S., Gay, D., Polastre, J., Szewczyk, R., Woo, A., ... \& Culler, D. E. (2004, March). The Emergence of Networking Abstractions and Techniques in TinyOS. In NSDI (Vol. 4, pp. 1-1).

Mamatha, G. S., \& Sharma, D. S. (2010). Analyzing the Manet Variations, Challenges, Capacity and Protocol Issues. International Journal of Computer Science \& Engineering Survey (IJCSES), 1(1), 14-21.

Manjeshwar, A., \& Agrawal, D. P. (2001, April). TEEN: ARouting Protocol for Enhanced Efficiency in Wireless Sensor Networks. In IPDPS (Vol. 1, p. 189).

Manjeshwar, A., \& Agrawal, D. P. (2002, April). APTEEN: A Hybrid Protocol for Efficient Routing and Comprehensive Information Retrieval in Wireless Sensor Networks. In ipdps (Vol. 2, p. 48).

Nasipuri, A. (2004). Mobile Ad Hoc Networks. Handbook of RF and Wireless Technologies, 59-100.

Qi, H., Kuruganti, P. T., \& Xu, Y. (2002). The development of localized algorithms in wireless sensor networks. Sensors, 2(7), 286-293.

Rashed, M., Kabir, M. H., Rahim, M. S., \& Ullah, S. E. (2012). Cluster based hierarchical routing protocol for wireless sensor network. arXiv preprint arXiv:1207.3876.

Sarrafzadeh, M., Dabiri, F., Jafari, R., Massey, T., \& Nahapetan, A. (2006, October). Low power light-weight embedded systems. In Low Power Electronics and Design, 2006. ISLPED'06. Proceedings of the 2006 International Symposium on (pp. 207-212). IEEE.

Schurgers, C., \& Srivastava, M. B. (2001). Energy efficient routing in wireless sensor networks. In Military Communications Conference, 2001. MILCOM 2001. Communications for NetworkCentric Operations: Creating the Information Force. IEEE (Vol. 1, pp. 357-361). IEEE.

Shah, R. C., \& Rabaey, J. M. (2002, March). Energy aware routing for low energy ad hoc sensor networks. In Wireless Communications and Networking Conference, 2002. WCNC2002. 2002 IEEE (Vol. 1, pp. 350-355). IEEE.

Smaragdakis, G., Matta, I., \& Bestavros, A. (2004). SEP: A stable election protocol for clustered heterogeneous wireless sensor networks. Boston University Computer Science Department.

Sun, J. Z. (2001). Mobile ad hoc networking: an essential technology for pervasive computing. In Info-tech and Info-net, 2001. Proceedings. ICII 2001-Beijing. 2001 International Conferences on (Vol. 3, pp. 316-321). IEEE.

Tilak, S., Abu-Ghazaleh, N. B., \& Heinzelman, W. (2002). A taxonomy of wireless micro-sensor network models. ACM SIGMOBILE Mobile Computing and Communications Review, 6(2), 28-36.

Yu, Y., Govindan, R., \& Estrin, D. (2001). Geographical and energy aware routing: A recursive data dissemination protocol for wireless sensor networks. Technical report ucla/csd-tr-01-0023, UCLA Computer Science Department.

Zadeh, L. A. (1965). Fuzzy sets. Information and control, 8(3), 338-353. 
1132

Zhao, J., \& Erdogan, A. T. (2006, June). A novel self-organizing hybrid network protocol for wireless sensor networks. In Adaptive Hardware and Systems, 2006. AHS 2006. First NASA/ESA Conference on (pp. 412-419). IEEE.

Zhang, Y., \& Lee, W. (2000, August). Intrusion detection in wireless ad-hoc networks. In Proceedings of the 6th annual international conference on Mobile computing and networking (pp. 275-283). ACM.

Zhang, Y., Yang, L. T., \& Chen, J. (Eds.). (2010). RFID and sensor networks: architectures, protocols, security, and integrations. CRC Press. 